\title{
Fabrication of high frequency nanometer scale mechanical resonators from bulk Si crystals
}

\author{
A. N. Cleland ${ }^{\text {a) }}$ and M. L. Roukes \\ Condensed Matter Physics 114-36, California Institute of Technology, Pasadena, California 91125
}

(Received 21 June 1996; accepted 22 August 1996)

\begin{abstract}
We report on a method to fabricate nanometer scale mechanical structures from bulk, single-crystal Si substrates. A technique developed previously required more complex fabrication methods and an undercut step using wet chemical processing. Our method does not require low pressure chemical vapor deposition of intermediate masking layers, and the final step in the processing uses a dry etch technique, avoiding the difficulties encountered from surface tension effects when wet processing mechanically delicate or large aspect ratio structures. Using this technique, we demonstrate fabrication of a mechanical resonator with a fundamental resonance frequency of $70.72 \mathrm{MHz}$ and a quality factor of $2 \times 10^{4}$. (C) 1996 American Institute of Physics. [S0003-6951(96)04144-7]
\end{abstract}

The combination of electron beam lithography and $\mathrm{Si}$ micromachining techniques makes it possible to fabricate submicron mechanical structures from single crystal substrates. Structures can be fabricated with fundamental mechanical resonance frequencies reaching into the microwave frequency bands, and can be made small enough that it is statistically unlikely that there are any crystalline defects contained within the structure. Such structures should exhibit very high quality factors and other mechanical properties that reflect the true nature of the material, such as large breaking stress. Elsewhere we have shown that such devices can be used in a new class of particle and energy sensors due to their very small mass, small size, high operating frequencies, and sensitivity to external conditions. ${ }^{1}$ Furthermore, a resonator with a fundamental mechanical resonance frequency of a few times $10^{9} \mathrm{~Hz}$ can be fabricated; this could exhibit macroscopic quantum effects at dilution refrigerator temperatures and might display interesting interactions with thermal phonons with the same range of frequencies. ${ }^{1}$

Other authors have reported recipes for fabricating submicron suspended Si structures. ${ }^{2,3}$ These recipes are somewhat more complicated and require more extensive processing equipment than the method we describe here. The final undercut step of the suspended structures is performed in our recipe by using a dry etch process, which avoids potential damage due to surface tension encountered in wet etch processes. We first describe the method used to fabricate suspended structures, and then describe the method used to measure the resonance properties of simple doubly clamped beams and show data for one such structure.

We used $\langle 100\rangle$ orientation, nominally undoped $n$-type $\mathrm{Si}$ wafers with a resistivity at $298 \mathrm{~K}$ of $>1000 \Omega \mathrm{cm}$. The substrates were cleaned, and on them a $1 \mu \mathrm{m} \mathrm{SiO}_{2}$ layer was grown by pyrogenic steam oxidation for $1 \mathrm{~h}$ at $1100{ }^{\circ} \mathrm{C}$, with $\mathrm{O}_{2}$ flowing at $0.4 \mathrm{scfm}$ through water at $95{ }^{\circ} \mathrm{C}$; this oxide layer serves as a mask for the isotropic reactive ion etching (RIE) of the Si substrate, the step that undercuts the suspended structures.

Large area contact pads were defined by optical lithography. A 100-nm-thick layer of $\mathrm{Ni}$ was rf sputtered onto a

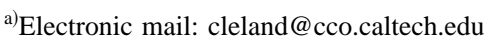

liftoff pattern, and the photoresist removed by soaking in acetone.

We next deposited a polymethyl methacrylate (PMMA) bilayer on the oxidized substrates, with a 400-nm-thick underlayer of 496 KD PMMA and a 100-nm-thick overlayer of 950 KD PMMA. We patterned the bilayer by electron beam lithography with a $40 \mathrm{kV}$ accelerating voltage and a dose of $350 \mu \mathrm{C} / \mathrm{cm}^{2}$. A 100-nm-thick layer of Ni was then rf sputter deposited onto the bilayer and then lifted off; the substrates then had the appearance of that shown in cross section in Fig. 1(a).

The pattern in the metal mask was transferred to the oxide layer beneath it by anisotropic RIE in a parallel plate reactor $^{4}$ that has a 6-in.-diam cathode, a 4 in. anode-tocathode spacing, and operates at $13.56 \mathrm{MHz}$. The cathode plate is water cooled to a little below room temperature. The etch chamber is oil diffusion pumped, and was always pumped to less than $2 \times 10^{-5}$ Torr. For oxide etching, we used $\mathrm{C}_{2} \mathrm{~F}_{6}$ flowing at $5 \mathrm{sccm}$ with an indicated chamber pressure of $60 \mathrm{mTorr}$. The chamber pressure was measured by a thermocouple-type gauge. ${ }^{5}$ We used a rf power of $50 \mathrm{~W}$, developing a plasma voltage of $280 \mathrm{~V}$ to the cathode of the reactor. The etch rate of $\mathrm{SiO}_{2}$ in these conditions was 180$210 \mathrm{~nm} / \mathrm{min}$, and was stopped by timing the etch duration; we found that $\mathrm{C}_{2} \mathrm{~F}_{6}$ did not etch $\mathrm{Si}$ at a noticeable rate, so the timing was not critical. The sidewalls in the patterned oxide were quite vertical, with an angle at most $5^{\circ}$ from normal. At this point, the substrates had the appearance of that shown in Fig. 1(b).

After removing the substrates for inspection, they were returned to the RIE chamber for anisotropic etching of the Si.

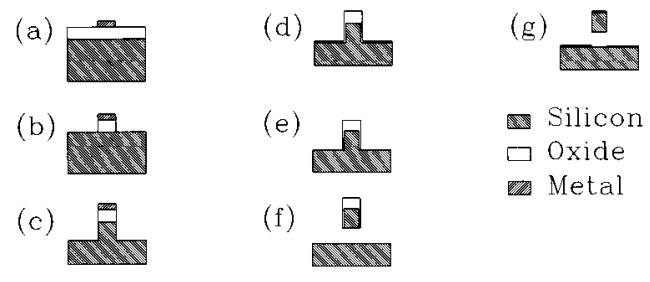

FIG. 1. (a)-(g) Schematic diagrams of the processing steps to generate suspended Si structures. The steps are as described. 


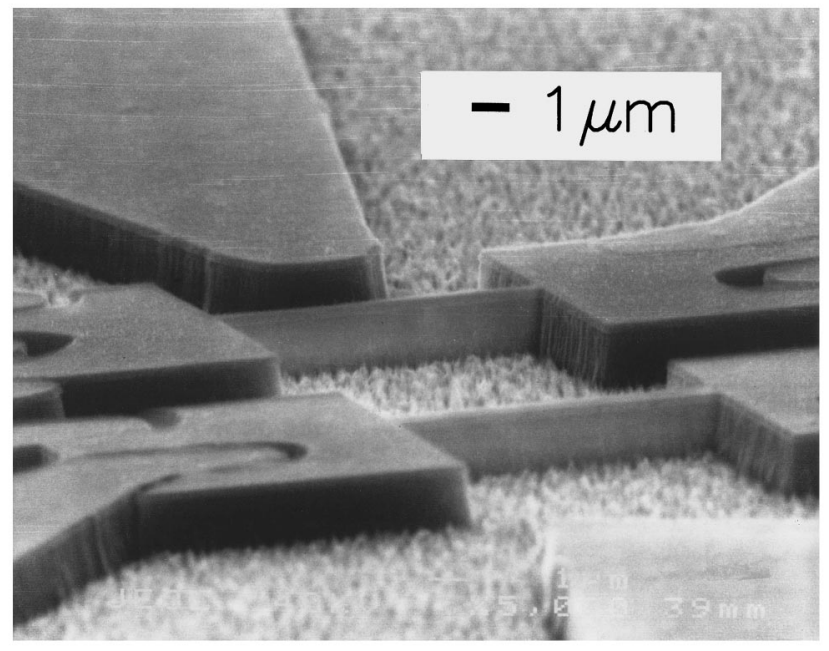

FIG. 2. SEM micrograph of the structures following the anisotropic Si etch.

We used a combination of $\mathrm{NF}_{3}$ and $\mathrm{CCl}_{2} \mathrm{~F}_{2}$, each flowing at $5 \mathrm{sccm}$ with a chamber pressure of $30 \mathrm{mTorr}$; $\mathrm{rf}$ power was $150 \mathrm{~W}$, with a plasma voltage of $420 \mathrm{~V}$. The etch rate for $\mathrm{Si}$ was found to be $1100 \mathrm{~nm} / \mathrm{min}$, with quite vertical sidewalls. At this point the structures were as shown in Fig. 1(c); a scanning electron microscope (SEM) micrograph of these structures is shown in Fig. 2. The Ni mask was removed by wet chemical etching and rinsing in de-ionized (DI) water. We then grew a sidewall oxide mask by pyrogenic oxidation at $1000{ }^{\circ} \mathrm{C}$ for $20 \mathrm{~min}$; see Fig. 1(d).

In order to remove the oxide layer on the base surface of the etched structure, the substrates were placed in the RIE chamber and etched using the anisotropic $\mathrm{SiO}_{2}$ recipe; the etch time was $15 \mathrm{~min}$, somewhat overetching to ensure that all the base layer $\mathrm{SiO}_{2}$ was removed.

At this point, the substrates had vertical ribs of singlecrystal $\mathrm{Si}$, with a thick oxide layer on the upper surface and a thin sidewall oxide on the vertical sidewalls; see Fig. 1(e). The vertical ribs were undercut in the final step, which was performed by RIE using pure $\mathrm{NF}_{3}$; etching was masked by the remaining oxide layers. The $\mathrm{NF}_{3}$ flow rate was $5 \mathrm{sccm}$, and the chamber pressure was $10 \mathrm{mTorr}$; rf power was 150 $\mathrm{W}$, with a plasma voltage of $450 \mathrm{~V}$. Because the etch rate of the top layer of $\mathrm{SiO}_{2}$ was nearly as rapid as the etch rate of $\mathrm{Si}$, timing in this step was critical. The structures were examined by SEM to ensure complete undercutting; see Fig. 3. Note that the undercut is not flat under the structure, but has a sharp point in the middle of the structure due to the isotropic nature of the etch; furthermore, we always observed some scalloping along the length of the structure, probably due to variations in oxidation of the exposed $\mathrm{Si}$ surface. Use of a non-RIE based etch ${ }^{6}$ might improve the surface quality.

At this point the structures were complete. The oxide layers could be removed by wet etching in hydrofluoric acid $(48 \%)$ and rinsing in de-ionized water. Metallization was also performed at this point, by thermal evaporation of an adhesion layer of $\mathrm{Cr}(5-10 \mathrm{~nm})$ followed by a layer of $\mathrm{Au}$ $(100 \mathrm{~nm})$. The undercutting of the entire structure ensured that the Au evaporated on the top of the structures was electrically isolated from that deposited in the base of the substrates; see Fig. 1(g). Typical isolation resistances, due to the

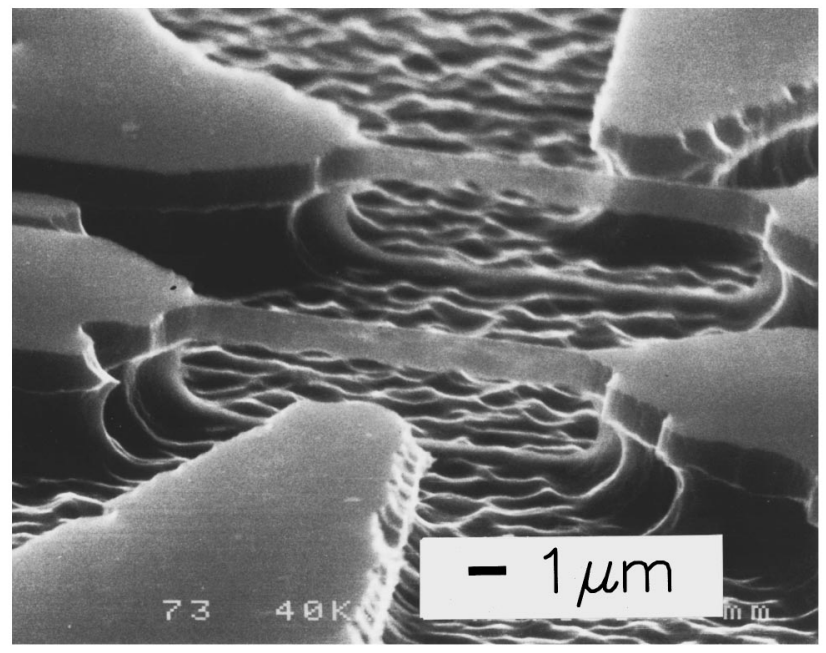

FIG. 3. SEM micrograph of an undercut Si beam, with length of $7.7 \mu \mathrm{m}$, width of $0.33 \mu \mathrm{m}$, and height of $0.8 \mu \mathrm{m}$. Some scalloping is visible along the bottom edge of the beam.

metal-Si Schottky barrier, were greater than $1 \mathrm{M} \Omega$ for bias voltages of less than about $0.1 \mathrm{~V}$.

Using the technique described above, we have fabricated a number of suspended $\mathrm{Si}$ beams and have measured their resonance properties at a temperature of $4.2 \mathrm{~K}$. The beams were placed in vacuum, with the long axis of the beam perpendicular to a magnetic field generated by a superconducting solenoid. Electrical connections were made by $\mathrm{Au}$ wire bonds to a chip carrier, and short lengths of $\mathrm{Cu}$ wire were soldered between the chip carrier and the center and the ground of a stainless steel coaxial cable that carried signals to the room temperature electronics. A network analyzer was used to drive an alternating current along the length of the beam and measure the response of the beam. The alternating current, transverse to the magnetic field, generates a Lorentz force that drives the beam transverse to its length and to the field direction. The motion of the beam then generates an electromotive force (EMF) along the length of the beam; the EMF is detected by the network analyzer. A schematic of the measurement setup is shown in Fig. 4. A similar technique has been used by other authors. ${ }^{7}$ Several authors have reported measurements on larger beams at lower frequencies performed using either electrostatic ${ }^{8}$ or optical ${ }^{9}$ detection, but these techniques do not extend easily to smaller structures at higher frequencies.

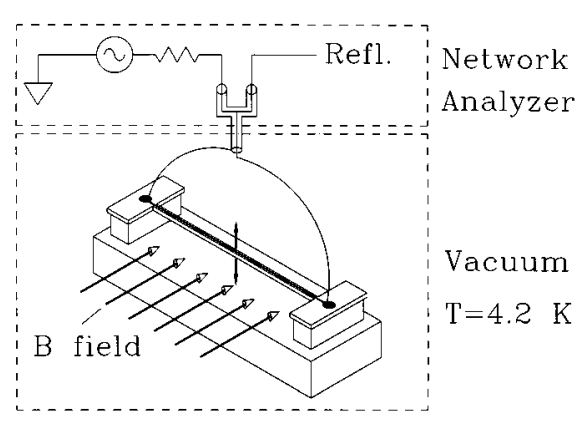

FIG. 4. Schematic illustration of the experimental setup used to measure the resonance properties of the $\mathrm{Si}$ structures. 


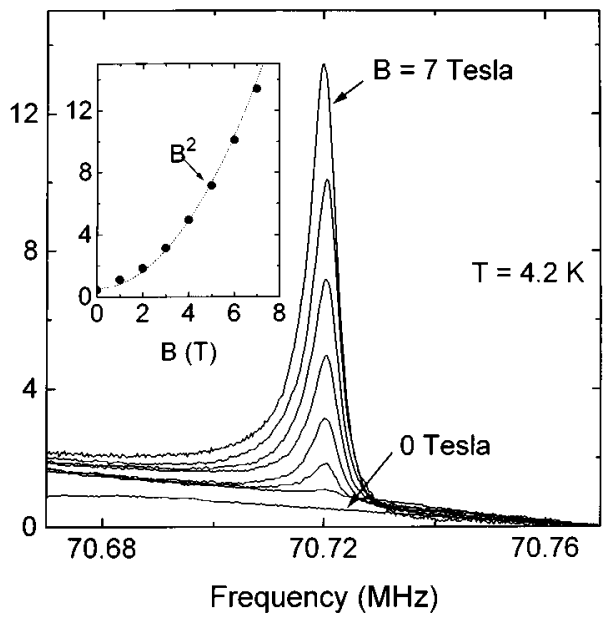

FIG. 5. Induced EMF as a function of drive frequency for the structure in Fig. 3. Magnetic field strength ranged from 0 . to $7 \mathrm{~T}$ at a temperature of 4.2 K. Drive amplitude was $10 \mathrm{mV}$. Inset: Induced EMF at resonance as a function of field strength. The vertical axis has same units as the main plot.

These beams should have mechanical resonance frequencies $\omega_{n}$ as given by

$$
\frac{\omega_{n}}{2 \pi}=C_{n} \sqrt{\frac{E}{\rho}} \frac{w}{L^{2}},
$$

where $E$ is Young's modulus, $\rho$ is the density, $w$ is the beam width in the direction of motion, and $L$ is the beam length; the constants $C_{n}$, of order unity, depend on the mode number $n$ and on the clamping conditions at the ends of the beam. If such a beam is driven by a force $F(\omega)$ near a resonance frequency $\omega_{n}$, then to first order in the mode amplitude the beam responds as a harmonic oscillator and, assuming a damping term proportional to the beam velocity, the amplitude $u(\omega)$ should have a Lorentzian line shape given in

$$
u(\omega)=\frac{F(\omega) / k_{\mathrm{eff}}}{\sqrt{\left(\omega^{2}-\omega_{n}^{2}\right)^{2}-\omega^{2} \omega_{n}^{2} / Q^{2}}},
$$

where $k_{\text {eff }}$ is the effective spring constant and $Q$ is the quality factor. Note that in our system the force $F$ depends linearly on the magnetic induction $B$, and the EMF generated by the motion is proportional to the product of $B$ and the beam velocity $\mathrm{d} u / \mathrm{d} t$, and should scale as $B^{2}$.

We have measured the fundamental resonance frequencies of a number of beams with different geometries, with resonance frequencies ranging from $400 \mathrm{kHz}$ to $120 \mathrm{MHz}$, and quality factors from about $10^{3}$ to a few times $10^{4}$. In Fig. 5(a) we display the measured resonance of the beam shown in Fig. 3 that was measured for a range of magnetic fields from 0 to $7 \mathrm{~T}$; the resonance frequency was $70.72 \mathrm{MHz}$, and the quality factor, determined by fitting a Lorentzian to the resonance shape, was found to be about $1.8 \times 10^{4}$. Shown in Fig. 5(b) is the peak height of the resonance measured as a function of the magnetic field induction; the induced EMF scales as the square of the field strength, as expected.

Note that the vertical scales of the plots in Fig. 5 cannot be interpreted to give beam displacements, as we do not know the exact coupling parameters of the beam to the coaxial cable used to transmit the radio-frequency signals. However, we estimate that the maximum displacement amplitudes for the data shown in Fig. 5 are a few nanometers. Larger drive currents were also used; they generated very noticeable divergences of the resonance shapes from the Lorentzian, as was expected from terms of higher order than the amplitude squared in the beam bending energy.

It would be of great interest to fabricate a $1 \mathrm{GHz}$ resonant beam using this technique by simply reducing the length $L$ of the beam by a factor of 4 . However, this would reduce the signal-to-noise $(\mathrm{S} / \mathrm{N})$ ratio of the measurement technique by a factor of at least 64 , and more if the $Q$ is adversely affected by the reduction in aspect ratio $L / w$. We propose fabrication of a beam with dimensions $1.3 \times 0.15$ $\times 0.2 \mu \mathrm{m}^{3}$, with an expected $\mathrm{S} / \mathrm{N}$ reduction of about 10 if the $Q$ remains the same. A reduction in the length and width of the beam of this order is fairly straightforward, while reducing the height would require very careful timing of the $\mathrm{Si}$ anisotropic etch. Use of laser end-point detection during RIE should make this feasible.

In conclusion, we report on a method for fabricating submicron single-crystal mechanical resonators that uses a single metal mask, two steps of thermal oxidation, and four steps of reactive ion etching to generate a suspended mechanical structure. We have also shown data taken on a radio frequency resonator at low temperatures, with a reasonably high quality factor. Work is in progress to fabricate resonators with higher fundamental resonance frequencies, and we are investigating methods to reach even higher quality factors.

The authors acknowledge Dr. Axel Scherer for many valuable conversations, and for use of his reactive ion etch system. This work was supported by ARPA under Contract No. DABT63-95-C-0112.

${ }^{1}$ A. N. Cleland and M. L. Roukes (unpublished).

${ }^{2}$ S. C. Arney and N. C. MacDonald, J. Vac. Sci. Technol. B 6, 341 (1988).

${ }^{3}$ J. Yao, S. Arney, and N. C. MacDonald, J. Microelectromech. Sys. 1, 14 (1992).

${ }^{4}$ Materials Research Corp., Orangeburg, NY.

${ }^{5}$ Convectron gauge (series 275, Granville-Phillips Corp., Boulder, CO).

${ }^{6}$ H. F. Winters and J. W. Coburn, Surf. Sci. Rep. 14, 161 (1992).

${ }^{7}$ D. S. Greywall et al., Phys. Rev. Lett. 72, 2992 (1994); D. S. Greywall, B. Yurke, P. A. Busch, and S. Arney, Europhys. Lett. 34, 37 (1996).

${ }^{8}$ R. E. Mihailovich and N. C. MacDonald, Sens. Actuators A 50, 199 (1995).

${ }^{9}$ J. D. Zook et al., Sens. Actuators A 52, 92 (1996); D. W. Burns et al., Sens. Actuators A 48, 179 (1995). 